\title{
Mutual friction in helium II: a microscopic approach
}

\author{
H. M. Cataldo* \\ Departamento de Física, Facultad de Ciencias Exactas y Naturales, \\ Universidad de Buenos Aires and Consejo \\ Nacional de Investigaciones Científicas y Técnicas, \\ RA-1428 Buenos Aires, Argentina
}

\begin{abstract}
We develop a microscopic model of mutual friction represented by the dissipative dynamics of a normal fluid flow which interacts with the helical normal modes of vortices comprising a lattice in thermal equilibrium. Such vortices are assumed to interact with the quasiparticles forming the normal fluid through a pseudomomentum-conserving scattering Hamiltonian. We study the approach to equilibrium of the normal fluid flow for temperatures below $1 \mathrm{~K}$, deriving an equation of motion for the quasiparticle pseudomomentum which leads to the expected form predicted by the HVBK equations. We obtain an expression for the mutual friction coefficient $B$ in terms of microscopic parameters, which turns out to be practically independent of the vortex mass for values arising from diverse theories. By comparing our expression of $B$ with previous theoretical estimates, we deduce interesting qualitative features about the excitation of Kelvin modes by the quasiparticle scattering.
\end{abstract}

PACS numbers: 67.25.bf, 67.25.dk, 67.25.dt

Keywords: Mutual friction, normal fluid flow, vortex oscillations.

*Electronic address: cataldo@df .uba.ar; URL: http://www.df .uba.ar/users/cataldo 


\section{Introduction}

When a sufficiently fast rotating sample of liquid helium is cooled below the lambda temperature, all the rotation of the superfluid becomes concentrated in a uniform array of quantized vortex filaments parallel to the axis of rotation $[1,2]$. By contrast, the macroscopic superfluid velocity field, corresponding to spatial averages over regions large compared with the spacing between vortices, yields the usual configuration of solid body flow, $\mathbf{v}_{s}(\mathbf{r})=\Omega_{\text {rot }} \hat{\mathbf{z}} \times \mathbf{r}$ for a rotation frequency $\Omega_{\text {rot }}$ around the $z$ axis. Just as the superfluid flow is microscopically formed by vortices, the normal fluid consists of superfluid quasiparticle excitations, phonons and rotons, the average flow of which is characterized by the normal fluid velocity field $\mathbf{v}_{n}$. Both fluids must move in equilibrium at the same velocity, and such a behavior is caused by the mutual friction force [3]. A well-known phenomenological model for this macroscopic dynamics is represented by the so-called Hall-Vinen-Bekharevich-Khalatnikov (HVBK) equations $[3,4]$. There is a simple configuration which allows to show the basic features of this process, namely rectilinear flows of uniform vorticity $\nabla \times \mathbf{v}_{s}=2 \Omega_{\text {rot }} \hat{\mathbf{z}}$, which coincides with that of the rotational scheme [5]. The HVBK equations for such flows are very simple and read

$$
\rho_{n} \frac{\partial \mathbf{v}_{n}}{\partial t}=\mathbf{F}=-\rho_{s} \frac{\partial \mathbf{v}_{s}}{\partial t}
$$

where $\rho_{n}$ and $\rho_{s}$ denote the normal fluid and superfluid mass densities, respectively, and the mutual friction force $\mathbf{F}$ can be written for temperatures below $1 \mathrm{~K}$ as [5],

$$
\mathbf{F}=-B \rho_{n} \Omega_{\text {rot }}\left(\mathbf{v}_{n}-\mathbf{v}_{s}\right),
$$

being $B$ a dimensionless dissipative coefficient. Such a low temperature regime corresponds to $\rho_{n} \ll \rho_{s}$, which, according to (1), implies that the main time dependence 
should lie within the normal fluid velocity. This suggests that a suitable approach to the problem may consist in regarding the superfluid component as a thermal equilibrium heat bath which interacts with a nonequilibrium normal fluid flow. Keeping such a picture as our basic premise, we shall analyze in the present paper a microscopic model of mutual friction, which reproduces the main features of the above macroscopic dynamics, yielding an explicit expression of $B$ as a function of microscopic parameters.

The microscopic basis of mutual friction remains as one of the most intrincate problems of superfluidity. In such a context, theoretical approaches may strongly differ, even in significant questions such as the existence of a nondissipative component of the mutual friction force [6], which is absent from our modelling [5]. A better understanding of the microscopic principles governing mutual friction would also contribute to clarify important issues on the subject of quantum turbulence at finite temperature [7]. In fact, it is just the mutual friction force which accounts for the strong locking between superfluid and normal fluid along the turbulent cascade, where recent simulations have shown that the residual slip velocity $\mathbf{v}_{s}-\mathbf{v}_{n}$ plays a central role [8]. In addition, such simulations suggest that the cross-over between zero-temperature and finite temperature quantum turbulence occurs at a lower temperature than the usual estimation of $1 \mathrm{~K}$, hence partially placing the latter regime within the temperature range of the present investigation.

Another important source of controversy arises from the mass of quantized vortices. On the one hand, many works have considered it as a negligible parameter under the assumption that it should be equivalent to the hydrodynamic mass of a core of atomic dimensions [1]. Another theories, however, yield several orders of magnitude higher 
values for the vortex mass, casting doubt on models based on massless vortices [9]. Moreover, it has been argued that an unambiguous vortex mass may not exist, and that inertial effects in vortex dynamics may be scenario-dependent [10]. Finally, we should also mention that there have been conflicting results for the vortex mass in superconductors as well [11]. A possible way out to such uncertainties has been recently suggested based on the concept of pseudomomentum [5]. Our present study of the dissipative normal fluid dynamics will utilize the concept of pseudomomentum as an important tool, finding again results which are practically independent of the vortex mass for a wide range of values.

Our approach will consist in assuming a heat bath formed by a vortex lattice in thermal equilibrium, which interacts with a quasiparticle flow. The dominant contribution to the heat capacity of such a lattice should arise from the thermal excitation of helical waves, corresponding to effectively independent vortices [12]. The role of such oscillations in mutual friction has been scarcely treated in the literature. We are only able to mention a couple of papers $[13,14]$, that long ago reached the conclusion that the damping of vortex oscillations due to phonon scattering, should not modify appreciably the value of the friction coefficient calculated for a rigid vortex. The same conclusion was recently obtained for a high-frequency branch of helical waves, within a wider temperature range, including a roton-dominated regime [15].

In building a theory with massive vortices, one can readily make use of a close analogy with the well-known electrodynamical problem of a point charge subjected to magnetic and electric fields [16]. Particularly, the quantization of the theory, which greatly simplifies the treatment when the scattering excitation of vortex waves is taken into account [13], arises immediately from this analogy. Such an analogy also leads 
to an immediate identification of the vortex pseudomomentum, allowing us to build a proper form for a pseudomomentum-conserving scattering Hamiltonian.

This paper is organized as follows. In the next section, we sketch out our derivation of the equation of motion for the normal fluid flow. In Sec. 3 we analyze the expression obtained for the mutual friction coefficient $B$ and in Sec. 4 we summarize our main results.

\section{Equation of motion for the normal fluid flow}

We assume vortex filaments performing helical oscillations about their unperturbed positions parallel to the $z$ axis. The wavelength $\lambda$ is supposed to be much greater than the amplitude (radius of the helix), and to have a full description of the helix, one should also know the direction (right or left) of the helical deformation, or equivalently, the direction of the wave vector $k \hat{\mathbf{z}}(k= \pm 2 \pi / \lambda)$. Periodic boundary conditions over a length $L$ (vortex line length) along the $z$ axis determine the possible values of the wave vector as $k=2 \pi m / L$, where $m$ is an integer. The vortex core position $\mathbf{r}(z)$ may then be written as a summation over generalized two-dimensional coordinates $\mathbf{r}_{k}(z)$ associated to normal modes labeled by the wave vector $k \hat{\mathbf{z}}[12,13]$. The quantization of the vortex Hamiltonian arises straightforwardly from the electromagnetic analogy [16]. Since the vortex core parameter is assumed to be much less than the wavelength, we have $\omega_{k} \ll \Omega$, being $\omega_{k}$ the Kelvin wave frequency and $\Omega=\rho_{s} \kappa / m_{v}$ the cyclotron frequency, with $m_{v}$ the vortex mass per unit length and $\kappa$ the quantum of circulation

[1]. This in turn ensures that cyclotron and Kelvin modes become decoupled [15], 
yielding a Hamiltonian of oscillatory modes given by

$$
\sum_{k \neq 0} \hbar \Omega\left(\alpha_{k}^{\dagger} \alpha_{k}+\frac{1}{2}\right)+\hbar \omega_{k}\left(\beta_{k}^{\dagger} \beta_{k}+\frac{1}{2}\right)
$$

where $\alpha_{k}^{\dagger}\left(\beta_{k}^{\dagger}\right)$ denotes a creation operator of right (left) circular quanta.

The Hamiltonian of the $k=0$ modes, corresponding to rigid displacements of the vortex filament [5], can be exactly solved [16], yielding in the limit $\Omega_{\text {rot }} \ll \Omega$ the decoupling of cyclotron and translational modes:

$$
\hbar \Omega\left(\alpha_{0}^{\dagger} \alpha_{0}+\frac{1}{2}\right)+\frac{\hbar \Omega_{\mathrm{rot}}}{2}\left(\beta_{0}^{\dagger}-\beta_{0}\right)^{2}
$$

Particularly, the translational coordinate $\mathbf{r}_{0}=\sqrt{\frac{\hbar}{2 \rho_{s} \kappa L}}\left[\left(\beta_{0}^{\dagger}+\beta_{0}\right) \hat{\mathbf{x}}+i\left(\beta_{0}^{\dagger}-\beta_{0}\right) \hat{\mathbf{y}}\right]$ evolves according to the superfluid velocity field

$$
\dot{\mathbf{r}}_{0}=-2 \Omega_{\text {rot }} y_{0} \hat{\mathbf{x}}
$$

The vortex Hamiltonian is given by the sum of (3) and (4), and the normal fluid Hamiltonian, corresponding to a noninteracting quasiparticle gas, reads $\sum_{\mathbf{q}} \hbar \omega_{\mathbf{q}} a_{\mathbf{q}}^{\dagger} a_{\mathbf{q}}$, where $a_{\mathbf{q}}^{\dagger}$ denotes a creation operator of quasiparticle excitations of pseudomomentum $\hbar \mathbf{q}$ and frequency $\omega_{\mathbf{q}}$. The interaction Hamiltonian between vortex and quasiparticles is represented by the pseudomomentum-conserving form:

$$
H_{\mathrm{int}}=\sum_{\mathbf{p}, \mathbf{q}} \int_{0}^{L} d z \Lambda_{\mathbf{p q}} a_{\mathbf{p}}^{\dagger} a_{\mathbf{q}} \exp \left[-i(\mathbf{p}-\mathbf{q}) \cdot \mathbf{r}(z)-i\left(p_{z}-q_{z}\right) z\right]
$$

where the parameters $\Lambda_{\mathbf{p q}}$ in (6) represent scattering amplitudes depending on wave vectors of scattered quasiparticles. The vortex pseudomomentum per unit length [5], integrated along the vortex line yields the generator of vortex translations or vortex pseudomomentum $-\rho_{s} \kappa L \hat{\mathbf{z}} \times \mathbf{r}_{0}$, which involves only translational coordinates, as expected. Then, adding such a pseudomomentum to the quasiparticle pseudomo- 
mentum $\sum_{\mathbf{q}} \hbar \mathbf{q} a_{\mathbf{q}}^{\dagger} a_{\mathbf{q}}$, we have the total pseudomomentum, which can be shown to commute with $H_{\text {int }}$.

The interaction Hamiltonian (6) is difficult to deal with, so recalling the low amplitude of the helical oscillations, we may rewrite the exponential in (6) as

$$
\begin{aligned}
& \exp \left[-i(\mathbf{p}-\mathbf{q}) \cdot \mathbf{r}(z)-i\left(p_{z}-q_{z}\right) z\right]= \\
& \exp \left[-i(\mathbf{p}-\mathbf{q}) \cdot \mathbf{r}_{0}\right] \exp \left[-i\left(p_{z}-q_{z}\right) z\right] \exp \left[-i(\mathbf{p}-\mathbf{q}) \cdot\left(\mathbf{r}(z)-\mathbf{r}_{0}\right)\right]
\end{aligned}
$$

and next approximate the last exponential to first order in $\mathbf{r}(z)-\mathbf{r}_{0}$. This procedure, however, is not valid for vortex modes with frequencies approaching zero, i.e. the lowest part of Kelvin's spectrum, as noted early by Fetter [13]. In fact, he showed that retaining a finite number of terms of such an exponential expansion leads to divergent results, analogous to those of the "infrared catastrophe" in electrodynamics. Here it is expedient to recall that within our study, each vortex forms part of a vortex lattice which will be regarded as a heat bath for the quasiparticle flow. Now, it is well known that rather simple models of heat bath often provide suitable descriptions of realistic environments [17]. Relying on this hypothesis and to overcome the above difficulty, we shall represent Kelvin's spectrum by a single frequency $w_{0}$, which will be eventually regarded as a temperature-dependent parameter in order to take into account the distinct features of the interaction of such waves with phonons and rotons. In summary, we shall make use of a simplified model of heat bath consisting of vortex modes of two frequencies $\left(w_{0} \ll \Omega\right)$ of opposite polarization. On the other hand, neglecting the vortex displacement in the $y$-direction [5], we shall replace the translational coordinate operator in the first exponential on the right-hand side of Eq. (7) by the $c$-number $\left\langle\mathbf{r}_{0}\right\rangle=-2 \Omega_{\text {rot }}\left\langle y_{0}\right\rangle t \hat{\mathbf{x}}$ (cf. Eq. (5)). Such a replacement becomes 
equivalent to having time-dependent scattering amplitudes in Eq. (6), i.e. with a time dependent phase factor, $\Lambda_{\mathbf{p q}} \exp \left[i\left(p_{x}-q_{x}\right) 2 \Omega_{\text {rot }}\left\langle y_{0}\right\rangle t\right]$. This factor, however, would not have any practical incidence, since our results will be shown to be dependent upon the absolute value of the scattering amplitudes. Finally, taking into account these approximations we may replace $\exp [-i(\mathbf{p}-\mathbf{q}) \cdot \mathbf{r}(z)] \simeq 1-i(\mathbf{p}-\mathbf{q}) \cdot\left(\mathbf{r}(z)-\mathbf{r}_{0}\right)$ in Eq. (6) yielding [15]

$$
\begin{aligned}
H_{\mathrm{int}} & =\sqrt{\frac{\hbar L}{2 \rho_{s} \kappa}} \sum_{k, \mathbf{p}, \mathbf{q}} \delta_{p_{z} q_{z}} \Lambda_{\mathbf{p q}} a_{\mathbf{p}}^{\dagger} a_{\mathbf{q}}\left\{\left[\left(q_{y}-p_{y}\right)+i\left(q_{x}-p_{x}\right)\right]\left[\alpha_{k}^{\dagger}+\left(1-\delta_{k 0}\right) \beta_{k}\right]\right. \\
& \left.+\left[\left(p_{y}-q_{y}\right)+i\left(q_{x}-p_{x}\right)\right]\left[\alpha_{k}+\left(1-\delta_{k 0}\right) \beta_{k}^{\dagger}\right]\right\}
\end{aligned}
$$

There is an additional parameter to be taken into account in our vortex heat bath, that is the total number of modes $2 L / \lambda_{\min }$. We shall assume for simplicity that both polarizations have a common 'ultraviolet' cutoff $\lambda_{\min }$, which should be greater than the vortex core parameter $(\sim 1 \AA)$ and the mean radius of the helix. Such a radius turns out to be of the order of the core parameter for cyclotron modes, while for Kelvin modes in a lattice of $\Omega_{\text {rot }} \sim 1 \mathrm{~s}^{-1}$ has been estimated [12] as $\sim 10^{3} \AA \sqrt{T / \mathrm{K}}$. So, we have assumed $\lambda_{\min } \sim 10^{3} \AA$ in our calculations.

To study the time evolution of the normal fluid, we have considered the dissipative dynamics of a quasiparticle flow which interacts with the heat bath formed by a uniform array of $N_{v}$ quantized vortex filaments. Then, following the methodology of Ref. [18], we have derived a system of non-Markovian equations ruling the time evolution of the quasiparticle populations $n_{\mathbf{q}}[19]$, which leads to the following equation of motion for the quasiparticle pseudomomentum:

$$
\begin{aligned}
& \sum_{\mathbf{q}} \hbar \mathbf{q} \dot{n}_{\mathbf{q}}=\frac{2 L^{2} N_{v}}{\rho_{s} \kappa \lambda_{\min }} \sum_{\mathbf{p}, \mathbf{q}, i}\left|\Lambda_{\mathbf{p q}}\right|^{2} \delta_{p_{z} q_{z}}(\mathbf{p}-\mathbf{q})(\mathbf{p}-\mathbf{q})^{2} \int_{0}^{t} d \tau \cos \left[\left(\omega_{p}-\omega_{q}+w_{i}\right) \tau\right] \\
& \times\left\{n_{\mathbf{q}}(t-\tau)\left[1+n_{\mathbf{p}}(t-\tau)\right]+\left[n_{\mathbf{q}}(t-\tau)-n_{\mathbf{p}}(t-\tau)\right]\left[e^{\hbar w_{i} / k_{B} T}-1\right]^{-1}\right\}
\end{aligned}
$$


where $w_{i}$ denotes the frequencies $\Omega$ and $w_{0}$. Assuming that the quasiparticle numbers in the above expression are well described by a local equilibrium form, the quasiparticle pseudomomentum $\sum_{\mathbf{q}} \hbar \mathbf{q} n_{\mathbf{q}}=A L \rho_{n}\left(\mathbf{v}_{n}-\mathbf{v}_{s}\right)$ should correspond to a macroscopically small area $A$ of the $x-y$ plane containing $N_{v}$ vortices, where the spatial dependence of the fields $\mathbf{v}_{n}$ and $\mathbf{v}_{s}$ can be neglected. In addition, given that the time dependence stems exclusively from $\mathbf{v}_{n}(t)$ (cf. Sec. 1), a straightforward calculation leads to the following non-Markovian equation:

$$
\dot{\mathbf{v}}_{n}=-\int_{0}^{t} d \tau\left[\mathbf{v}_{n}(t-\tau)-\mathbf{v}_{s}\right] \mu(\tau)
$$

with a memory kernel given by

$$
\begin{aligned}
\mu(\tau) & =\frac{L \hbar \Omega_{\mathrm{rot}}}{k_{B} T \rho_{n} \rho_{s} \kappa^{2} \lambda_{\min }} \sum_{\mathbf{p}, \mathbf{q}, i}\left|\Lambda_{\mathbf{p q}}\right|^{2} \delta_{p_{z} q_{z}} \cos \left[\left(\omega_{p}-\omega_{q}+w_{i}\right) \tau\right]\left\{|\mathbf{p}-\mathbf{q}|^{4} g_{+}\left(w_{i}\right)\right. \\
& \left.+\left[|\hat{\mathbf{z}} \times(\mathbf{p} \times \hat{\mathbf{z}})|^{4}-|\hat{\mathbf{z}} \times(\mathbf{q} \times \hat{\mathbf{z}})|^{4}\right] g_{-}\left(w_{i}\right)\right\} n\left(\omega_{q}\right)\left[1+n\left(\omega_{p}\right)\right]\left[1+n\left(w_{i}\right)\right],(11)
\end{aligned}
$$

where

$$
g_{ \pm}\left(w_{i}\right)=\frac{n\left(\omega_{p}\right)}{n\left(\omega_{q}-w_{i}\right)} \pm \frac{n\left(\omega_{q}\right) \exp \left[\hbar\left(\omega_{q}-\omega_{p}-w_{i}\right) / k_{B} T\right]}{n\left(\omega_{p}+w_{i}\right)}
$$

and $n(w)=\left[\exp \left(\hbar w / k_{B} T\right)-1\right]^{-1}$. In the thermodynamic limit, the summations over $\mathbf{p}$ and $\mathbf{q}$ in (11) become integrals and $\mu(\tau)$ acquires a finite lifetime. If such a lifetime can be regarded as microscopic in comparison with the observational timescale, equation (10) may be transformed according to the Markov approximation into the differential equation:

$$
\dot{\mathbf{v}}_{n}=-\nu\left[\mathbf{v}_{n}(t)-\mathbf{v}_{s}\right]
$$

with

$$
\begin{aligned}
\nu & =\int_{0}^{\infty} \mu(\tau) d \tau=\frac{L h \Omega_{\mathrm{rot}}}{k_{B} T \rho_{n} \rho_{s} \kappa^{2} \lambda_{\min }} \sum_{\mathbf{p}, \mathbf{q}, i}\left|\Lambda_{\mathbf{p q}}\right|^{2} \delta_{p_{z} q_{z}} \delta\left(\omega_{p}-\omega_{q}+w_{i}\right) \\
& \times|\mathbf{p}-\mathbf{q}|^{4} n\left(\omega_{q}\right)\left[1+n\left(\omega_{p}\right)\right]\left[1+n\left(w_{i}\right)\right],
\end{aligned}
$$


where the continuum limit corresponds to the replacement $\sum_{\mathbf{p}, \mathbf{q}} \delta_{p_{z} q_{z}} \rightarrow$ $\left[A^{2} L /(2 \pi)^{5}\right] \int d^{3} \mathbf{p} \int d^{3} \mathbf{q} \delta\left(p_{z}-q_{z}\right)$. Actually, we have studied the non-Markovian equation (10) finding that memory effects are negligible for $\Omega_{\text {rot }} \ll w_{0}$ [19], which will be assumed hereafter. Finally, taking into account (13), (1) and (2), we may obtain the expression of the mutual friction parameter from $B=\nu / \Omega_{\mathrm{rot}}$.

\section{Study of the mutual friction parameter $B$}

An explicit expression for the friction parameter $B$ can be extracted by computing the right-hand side of Eq. (14), which is reduced to the single one-dimensional integral $[20]:$

$B=\frac{19 \hbar^{3}}{70(2 \pi)^{2} c_{s}^{2} \rho_{n} \rho_{s} k_{B} T \lambda_{\min }} \sum_{i}\left[1+n\left(w_{i}\right)\right] \int_{0}^{\infty} d p\left|\omega_{p}^{\prime}\right| n\left(\omega_{p}+w_{i}\right)\left[1+n\left(\omega_{p}\right)\right] \sum_{j} \Gamma\left(p, q_{j}^{(i)}\right)$,

where $\omega_{p}^{\prime}\left(c_{s}\right)$ denotes the quasiparticle group (sound) velocity and

$$
\Gamma(p, q)=\left\{\begin{array}{cc}
p^{2} q\left(q^{4}+p^{4} / 5+2 p^{2} q^{2}\right) & (p \leq q) \\
q^{2} p\left(p^{4}+q^{4} / 5+2 p^{2} q^{2}\right) & (q \leq p),
\end{array}\right.
$$

being $q=q_{j}^{(i)}$ the roots of the equation $\omega_{q}=\omega_{p}+w_{i}$. In obtaining (15) we have utilized the expression of the scattering amplitude given in ref. [20], which has been shown to lead to a very good agreement with the experimental determinations of the longitudinal friction coefficient $D$ for temperatures below $1 \mathrm{~K}$ [18]. From (15) we may see that $B$ consists of two terms arising from the frequencies $w_{i}=w_{0}$ and $w_{i}=\Omega$. Such contributions, however, are weighted by respective factors $\left[1+n\left(w_{0}\right)\right] \gg[1+n(\Omega)]$, so the cyclotron contribution will be always negligible with respect to that arising from the frequency $w_{0}$ and we have that, in practice, $B$ will correspond to the limit 
of massless vortices, $\Omega \rightarrow \infty$. The expression (15) leads to simpler phonon and roton approximations. If we restrict ourselves to $w_{0} \lesssim 10^{10} \mathrm{~s}^{-1}$, such a frequency may be neglected everywhere in $(15)$, except in the factor $\left[1+n\left(w_{0}\right)\right]$. Then, to approximate for phonon temperatures $(\mathrm{T}<0.4 \mathrm{~K})$, we use the linear dispersion relation $\omega_{p}=c_{s} p$ and get

$$
B_{\mathrm{ph}}=\frac{254.9\left[1+n\left(w_{0}\right)\right]\left(k_{B} T\right)^{3}}{\hbar^{2} c_{s}^{4} \rho_{s} \lambda_{\min }} .
$$

On the other hand, for temperatures above $0.6 \mathrm{~K}$, only the portion of the dispersion curve around the roton minimum makes a significant contribution to the integrand in (15), then making use of the usual approximations in roton calculations [20], we obtain

$$
B_{\mathrm{r}}=\frac{2.079\left[1+n\left(w_{0}\right)\right] \hbar k_{0}^{3} \sqrt{k_{B} T}}{c_{s}^{2} \sqrt{\mu} \rho_{s} \lambda_{\min }},
$$

where $\mu$ and $k_{0}$ are parameters entering the Landau parabolic approximation, $\omega_{p}=$ $\Delta / \hbar+\hbar\left(p-k_{0}\right)^{2} / 2 \mu$.

Experimental determinations of $B$ have been reported only above $1.3 \mathrm{~K}$. However, we may utilize the following expression valid for temperatures below $1 \mathrm{~K}$ [1],

$$
B=\frac{2 D}{\rho_{n} \kappa}
$$

and replace $D$ in (19) by means of the Iordanskii theoretical estimate for the phonon temperature range [21], yielding

$$
B_{\mathrm{ph}}=8.17 \frac{k_{B} T}{m_{4} c_{s}^{2}}
$$

On the other hand, for the roton temperature range, we may replace $D=\rho_{n} v_{G} \sigma_{\|}$in (19) yielding,

$$
B_{\mathrm{r}}=\frac{2 \sigma_{\|}}{\kappa} \sqrt{\frac{2 k_{B} T}{\pi \mu}} \simeq 1.5 \sqrt{T} \mathrm{~K}^{-\frac{1}{2}}
$$


being $v_{G}$ the average group velocity of rotons and $\sigma_{\|} \simeq 8.38 \AA$ the roton scattering length $[1,2]$. Notice that we have replaced the friction coefficient $D$ by expressions corresponding to a straight vortex, since corrections due to vortex bending should be negligible, as seen in Sec. 1. This of course does not mean that vortices would remain straight against the quasiparticle scattering; on the contrary, thermal excitation of Kelvin waves is undoubtedly expected to occur, although details of this process are not evident from expressions (20) and (21). However, some features about such a process may be deduced from our results (17) and (18). First it is convenient to discuss the physical meaning of the frequency $w_{0}$. Since such a frequency is intended for representing the whole spectrum of Kelvin waves in the context of an interaction with quasiparticles, it should not be surprising to find that quite distinct values of $w_{0}$ could be required in order to better estimate interactions with phonons or rotons. This amounts to assuming a dependence of $w_{0}$ on temperature, which may be fully extracted by equating our results with the theoretical expressions (20) and (21). Then, from (17) and (20) we may conclude that phonon scattering at a temperature $T$ should be expected to excite Kelvin waves about a representative frequency given by

$$
w_{0} \simeq 5.64 \times 10^{8} \mathrm{~s}^{-1} \mathrm{~K}^{-3} T^{3}
$$

while (18) and (21) imply that roton scattering should be likely to excite Kelvin waves about frequency

$$
w_{0} \simeq 2.08 \times 10^{10} \mathrm{~s}^{-1} \mathrm{~K}^{-1} T \text {. }
$$

Recall that according to the Markov approximation one should assume $w_{0} \gg \Omega_{\text {rot }} \sim$ $1 \mathrm{~s}^{-1}$, which sets up a lower bound for the validity of the result (22) at temperatures above $\sim 0.01 \mathrm{~K}$. In addition, the assumption $\lambda_{\min } \sim 10^{3} \AA$ implies an upper bound 
for the Kelvin spectrum, $\max \left(\omega_{k}\right) \sim 10^{8} \mathrm{~s}^{-1}$, which turns out to be consistent with a phonon temperature range below $0.4 \mathrm{~K}$ in (22). However, the values arising from (23) seem to be overestimated for roton temperatures, since they would only be consistent with a $\lambda_{\text {min }}$ of order $10^{2} \AA$. In addition, such values of $\omega_{0}$ could reach the order of cyclotron frequencies arising from some theories of the vortex mass [9], contradicting the previous assumption $\omega_{k} \ll \Omega$. This suggests that only the qualitative trend $w_{0} \sim T$ should be taken into account from the result (23).

\section{Summary}

We have analyzed a microscopic model of mutual friction represented by the dissipative dynamics of a normal fluid flow, which interacts with the helical normal modes of vortices comprising a lattice in thermal equilibrium. Such vortices interact with the quasiparticles forming the normal fluid through a pseudomomentum-conserving scattering Hamiltonian. Assuming a simplified model for the vortex heat bath, we have derived an equation of motion for the quasiparticle pseudomomentum leading to the expected form predicted by the HVBK equations. We have shown that the mutual friction coefficient $B$ turns out to be practically independent of the values of vortex mass arising from diverse theories. Finally, from a comparison of our expression of $B$ with previous theoretical estimates, we have deduced interesting qualitative features about the interaction of quasiparticles with Kelvin modes, namely phonon (roton) scattering at a temperature $T$ should be expected to excite Kelvin waves about representative frequencies proportional to $T^{3}(T)$.

This work was supported by CONICET, Argentina through the grant PIP 5409. 
[1] R. J. Donnelly, Quantized Vortices in Helium II, Cambridge University Press, Cambridge (1991).

[2] C. F. Barenghi, R. J. Donnelly and W. F. Vinen, J. Low. Temp. Phys. 52, 189 (1983).

[3] H. E. Hall and W. F. Vinen, Proc. Roy. Soc. London Ser. A 238, 215 (1956).

[4] I. L. Bekharevich and I. M. Khalatnikov, Zh. Éksp. Teor. Fiz. 40, 920 (1961) [Sov. Phys. JETP 13, 643 (1961)].

[5] H. M. Cataldo, J. Phys. A: Math. Theor. 41, 295501 (2008).

[6] P. Ao and D. J. Thouless, Phys. Rev. Lett. 70, 2158 (1993); G. E. Volovik, Phys. Rev. Lett. 77, 4687 (1996); E. B. Sonin, Phys. Rev. B55, 485 (1997).

[7] Quantized Vortex Dynamics and Superfluid Turbulence, ed. by C. F. Barenghi, R. J. Donnelly and W. F. Vinen, Springer, Berlin (2001).

[8] P.-E. Roche, C. F. Barenghi and E. Leveque, http://arxiv.org/abs/0905.2754

[9] V. N. Popov, Zh. Éksp. Teor. Fiz. 64, 672 (1973) [Sov. Phys. JETP 37, 341 (1973)]; J. M. Duan, Phys. Rev. B49, 12381 (1994); J.-M. Tang, Intl. J. Mod. Phys. B15, 1601 (2001).

[10] D. J. Thouless and J. R. Anglin, Phys. Rev. Lett. 99, 105301 (2007).

[11] J. H. Han, J. S. Kim, M. J. Kim and P. Ao, Phys. Rev. B71, 125108 (2005).

[12] A. L. Fetter, Phys. Rev. 162, 143 (1967).

[13] A. L. Fetter, Phys. Rev. 186, 128 (1969).

[14] E. B. Sonin, Zh. Éksp. Teor. Fiz. 69, 921 (1975) [Sov. Phys. JETP 42, 469 (1976)].

[15] H. M. Cataldo, J. Phys. A: Math. Gen. 38, 7929 (2005). 
[16] D. Yoshioka, The Quantum Hall Effect, Springer, Berlin (2002), chap. 2.

[17] A. O. Caldeira and A. J. Leggett, Ann. Phys. (N.Y.) 149, 374 (1983); L. D. Chang and S. Chakravarty, Phys. Rev. B31, 154 (1985).

[18] H. M. Cataldo and D. M. Jezek, J. Low Temp. Phys. 136, 217 (2004).

[19] H. M. Cataldo, http://arxiv.org/abs/cond-mat/0604109

[20] H. M. Cataldo and D. M. Jezek, Phys. Rev. B65, 184523 (2002).

[21] S. V. Iordanskii, Zh. Éksp. Teor. Fiz. 49, 225 (1965) [Sov. Phys. JETP 22, 160 (1966)]. 